\title{
Discovery of thermonuclear (Type I) X-ray bursts in the X-ray binary Swift J1858.6-0814 observed with NICER and NuSTAR
}

\author{
Buisson, D J K; Altamirano, D; Bult, P; Mancuso, G C; Güver, T; Jaisawal, Gaurava Kumar; Hare, J; \\ Albayati, A C; Arzoumanian, Z; Segura, N Castro
}

Total number of authors:

25

Published in:

Monthly Notices of the Royal Astronomical Society

Link to article, DOI:

$10.1093 / \mathrm{mnras} / \mathrm{staa} 2749$

Publication date:

2020

Document Version

Peer reviewed version

Link back to DTU Orbit

Citation $(A P A)$ :

Buisson, D. J. K., Altamirano, D., Bult, P., Mancuso, G. C., Güver, T., Jaisawal, G. K., Hare, J., Albayati, A. C., Arzoumanian, Z., Segura, N. C., Chakrabarty, D., Gandhi, P., Guillot, S., Homan, J., Gendreau, K. C., Jiang, J., Malacaria, C., Miller, J. M., Arabac, M. Ö., ... Walton, D. J. (2020). Discovery of thermonuclear (Type I) X-ray bursts in the X-ray binary Swift J1858.6-0814 observed with NICER and NuSTAR. Monthly Notices of the Royal Astronomical Society, 499, 793-803. https://doi.org/10.1093/mnras/staa2749

\section{General rights}

Copyright and moral rights for the publications made accessible in the public portal are retained by the authors and/or other copyright owners and it is a condition of accessing publications that users recognise and abide by the legal requirements associated with these rights.

- Users may download and print one copy of any publication from the public portal for the purpose of private study or research.

- You may not further distribute the material or use it for any profit-making activity or commercial gain

- You may freely distribute the URL identifying the publication in the public portal 


\title{
Discovery of thermonuclear (Type I) X-ray bursts in the X-ray binary Swift J1858.6-0814 observed with NICER and NuSTAR
}

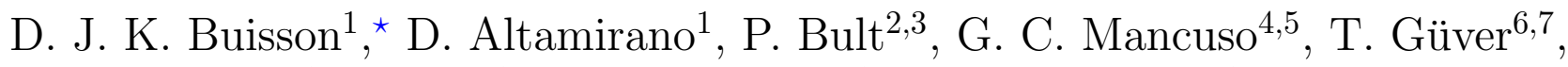 \\ G. K. Jaisawal ${ }^{8}$, J. Hare ${ }^{3} \dagger$, A. C. Albayati ${ }^{1}$, Z. Arzoumanian ${ }^{3}$, N. Castro Segura ${ }^{1}$, \\ D. Chakrabarty ${ }^{9}$, P. Gandhi ${ }^{1}$, S. Guillot ${ }^{10}$, J. Homan ${ }^{11,12}$, K. C. Gendreau ${ }^{3}$,
} J. Jiang ${ }^{13,14}$, C. Malacaria ${ }^{15,16} \ddagger$, J. M. Miller ${ }^{17}$, M. Özbey Arabac1 ${ }^{1,18}$, R. Remillard ${ }^{9}$, T. E. Strohmayer ${ }^{19}$, F. Tombesi ${ }^{2,20,3,21}$, J. A. Tomsick ${ }^{22}$, F. M. Vincentelli ${ }^{1}$ and D. J. Walton ${ }^{23}$ Affiliations at end.

Accepted XXX. Received YYY; in original form ZZZ

\begin{abstract}
Swift J1858.6-0814 is a recently discovered X-ray binary notable for extremely strong variability (by factors $>100$ in soft X-rays) in its discovery state. We present the detection of five thermonuclear (Type I) X-ray bursts from Swift J1858.6-0814, implying that the compact object in the system is a neutron star. Some of the bursts show photospheric radius expansion, so their peak flux can be used to estimate the distance to the system. The peak luminosity, and hence distance, can depend on several system parameters; for the most likely values, a high inclination and a helium atmosphere, $D=12.8_{-0.6}^{+0.8} \mathrm{kpc}$, although systematic effects allow a conservative range of $9-18 \mathrm{kpc}$. Before one burst, we detect a QPO at $9.6 \pm 0.5 \mathrm{mHz}$ with a fractional $\mathrm{rms}$ amplitude of $2.2 \pm 0.2 \%(0.5-10 \mathrm{keV})$, likely due to marginally stable burning of helium; similar oscillations may be present before the other bursts but the light curves are not long enough to allow their detection. We also search for burst oscillations but do not detect any, with an upper limit in the best case of $15 \%$ fractional amplitude (over $1-8 \mathrm{keV}$ ). Finally, we discuss the implications of the neutron star accretor and this distance on other inferences which have been made about the system. In particular, we find that Swift J1858.60814 was observed at super-Eddington luminosities at least during bright flares during the variable stage of its outburst.
\end{abstract}

Key words: accretion, accretion discs - stars: neutron - X-rays: binaries - X-rays: bursts

\section{INTRODUCTION}

A key aspect of accreting systems is the object onto which the accretion is occurring; in X-ray binaries (XRBs) this is either a neutron star (NS) or black hole (BH). Many observable properties are similar in either case, so determining which is present is often a challenging task.

There are severa/ properties which can divide NSs and BHs as populations and some features which empirically appear to occur in only one type of system. Firstly, outbursts of the different classes of source follow different tracks in gross

Email: d.j.k.buisson@soton.ac.uk

† NASA Postdoctoral Fellow

¥ NASA Postdoctoral Fellow properties such as the hardness-intensity or colour-colour diagrams (e.g. van der Klis 2006). However, this requires monitoring of the full outburst and some sources do not follow the typical patterns. Additionally, quasi-periodic oscillations (QPOs) are only found at $\mathrm{kHz}$ frequencies in neutron star systems (van der Klis et al. 1996; Strohmayer et al. 1996), although there is not yet a universally accepted model for their production (e.g. review by van der Klis 2006).

Also, BH and NS systems can be separated in the radio/X-ray luminosity plane (while in the hard state), with BH systems being radio brighter (Migliari \& Fender 2006; Gallo et al. 2018). Similarly, the hard Comptonised component tends to have a higher temperature in $\mathrm{BH}$ systems (Burke et al. 2017). However, the loci of BHs and NSs overlap in these properties, so they cannot be used to determine 
the accretor definitively in an individual source, particularly where a source shows unusual properties.

Other properties of an accreting system can give a definitive determination of whether the accreting object is a black hole or neutron star. To confirm a black hole accretor requires a dynamical mass measurement which is greater than possible neutron star masses (e.g. Webster \& Murdin 1972; Bolton 1972; Orosz \& Bailyn 1997), since there are no particular accretion properties which are unique to black holes. Conversely, there are several properties which are confirmed as unique to neutron stars, since the neutron star surface can provide an additional location for emission components and they can support large scale magnetic fields. The emission from this surface may be detected directly as a soft $(0.1-0.3 \mathrm{keV})$ blackbody-like component (e.g. Brown et al. 1998). This component is much fainter than the accretion luminosity, so cannot be identified during the first outburst in which a source is detected and requires sensitive observations to detect. Also, neutron stars can pulse coherently on their spin period, which can be observed at wavelengths from radio (Hewish et al. 1968) to X-ray (e.g. review by Patruno \& Watts 2012). A further feature of X-ray binaries particular to those hosting neutron stars is Type I X-ray bursts (e.g. Grindlay et al. 1976; Hoffman et al. 1978; Lewin et al. 1993; Strohmayer \& Bildsten 2006; Galloway et al. 2008a; Galloway \& Keek 2017).

Type I X-ray bursts occur due to explosive thermonuclear burning of accreted material on the neutron star surface. As material accretes onto the neutron star, it adds to layers of hydrogen and helium on the surface. When the pressure at the bases of these layers becomes large enough, it will ignite thermonuclear burning. Depending on the conditions, this burning may occur stably, contributing to the power in the persistent emission, or explosively, producing Type I bursts. These bursts can be ignited by hydrogen and/or helium (or occasionally carbon in 'superbursts', e.g. Cornelisse et al. 2000; Cumming \& Bildsten 2001; Strohmayer \& Brown 2002; in't Zand 2017), depending on the stability of each burning process, which depends principally on the accretion rate (Narayan \& Heyl 2003) but also the metallicity of the accreted material and the internal temperature of the neutron star (Bildsten 1995, 1998; Cumming \& Macbeth 2004). The basic classes of burning are thought to be as follows; while these classes of burning are reproduced in most numerical studies of Type I bursts, the exact values of accretion rate at which each one occurs differ between works (e.g. Fujimoto et al. 1981; Bildsten 1998; Narayan \& Heyl 2003).

At low accretion rates, a layer of hydrogen builds up before it reaches sufficient pressure to begin burning. At this point, the energy released by ignition causes most of the hydrogen layer to burn rapidly, which is observed as a Type I burst. At higher accretion rates, the accreted hydrogen promptly reaches sufficient temperature and pressure to burn so the burning is stable. When sufficient helium builds up, the pressure ignites helium burning which is responsible for the burst. At still higher accretion rates, not all of the hydrogen can be burnt before helium ignition occurs; this hydrogen is burnt along with the helium in a mixed burst. At the highest accretion rates, both hydrogen and helium burning occur stably so no Type I bursts

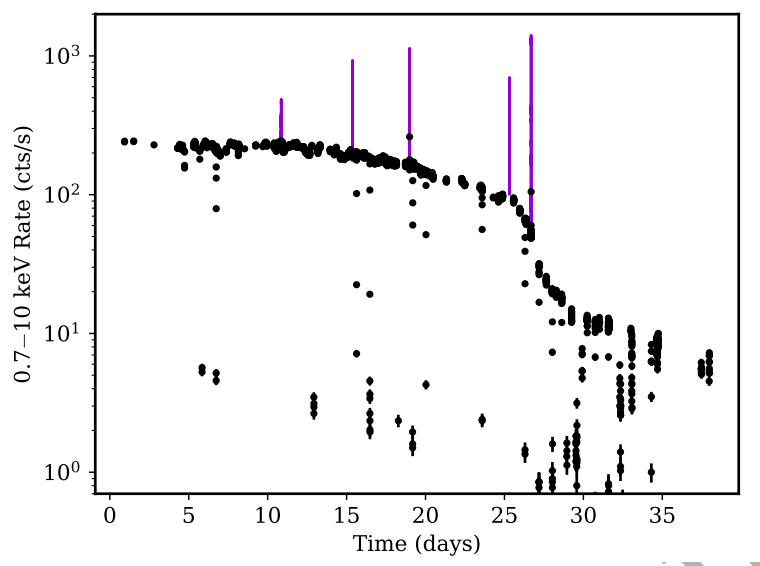

Figure 1. NICER light curve of Swift J1858.6-0814 since leaving Sun constraint in 2020, showing times of observed Type 1 bursts (purple). In addition to the long-term flux decrease, several dips and eclipses are visible; these will be considered in detail in future work. The full NICER light curve is shown in black at a resolution of $40 \mathrm{~s}$ and the bursts (purple) extend to their maximum count rate at $0.1 \mathrm{~s}$ resolution. The zero-point for the time axis is the start of 2020 February 25 (MUD 58904).

are observed. However, close to the transition to stability, the burning is marginally stable and has an oscillatory mode (Heger et al. 2007), which has been used to explain the millihertz QPOs observed prior to some Type I bursts (Revnivtsev et al. 2001; Altamirano et al. 2008; Lyu et al. 2016; Mancuso ét al. 2019).

There are also other effects which can affect the occurrence and type (fuel) of thermonuclear bursts. Each burst may not burn all of the available fuel, so some hydrogen and helium will remain after one burst and can affect properties of following bursts. Similarly, the burnt material will contain additional carbon, nitrogen and oxygen from helium burning. These nuclei catalyse hydrogen burning so can affect later bursts as well. The neutron star spin (Spitkovsky et al. 2002; Galloway et al. 2018) and the geometry of where on the star the material is accreted (Kajava et al. 2014) can also affect burst properties.

Type I bursts can sometimes be used as standard candles, as they can be bright enough to reach the Eddington limit. In this situation, the radiation pressure lifts material in the NS surface and the atmosphere expands in Photospheric Radius Expansion (PRE; Tawara et al. 1984; Lewin et al. 1984). Since the Eddington limit is only weakly dependent on radius, this produces a period during which the luminosity remains constant at the (known) Eddington value. This PRE phase may be identified (and distinguished from a simple plateau in the burning rate) by measuring the change in photospheric radius from the time-resolved X-ray spectrum. The measured flux during the PRE phase may then be used with this standard candle to estimate the distance to the source (van Paradijs 1978; Kuulkers et al. 2003).

\subsection{Swift J1858.6-0814}

The low-mass X-ray binary Swift J1858.6-0814 has been in its first observed outburst since late 2018 (Krimm et al. 
2018). The X-ray emission in the initial phase of the outburst was highly variable as was the emission in other wavebands (Ludlam et al. 2018; van den Eijnden et al. 2020, Fogantini et al. in prep.): the NICER $0.5-10 \mathrm{keV}$ count rate peaks at over $650 \mathrm{cts} / \mathrm{s}$ within $200 \mathrm{~s}$ of intervals at $\approx 2.5 \mathrm{cts} / \mathrm{s}$ (Fogantini et al. in prep.), much larger than the typical tens of percent RMS on these timescales (McClintock \& Remillard 2006). We refer to this stage of the outburst (all observations in 2018 and 2019) as the flaring state. The X-ray spectra were also extremely hard: $\Gamma<1$ if fitted with a simple powerlaw, (Kennea \& Krimm 2018; Ludlam et al. 2018), compared to typical $\Gamma>1.5$ (e.g. Zdziarski et al. 1999). This may be explained by the contribution of reflection and absorption: they also show a strong neutral iron $\mathrm{K} \alpha$ line and K edge (Reynolds et al. 2018; Hare et al. 2020) and soft X-ray emission lines (Buisson et al. 2020a). It also shows P-Cygni lines in its optical spectra, which look similar to those seen in several BH XRBs (Munoz-Darias et al. 2019; Muñoz-Darias et al. 2020, Castro-Segura in prep.), as well as strongly variable optical emission (Paice et al. 2018). These properties have led to Swift J1858.6-0814 being viewed (Hare et al. 2020) as an analogue of V404 Cyg (Gandhi et al. 2016; Walton et al. 2017; Motta et al. 2017) and V4641 Sgr (Wijnands \& van der Klis 2000; Revnivtsev et al. 2002), which have been dynamically confirmed as hosting black holes (Casares et al. 1992; Orosz et al. 2001, respectively). Swift J1858.6-0814 also lies within the range occupied by BHs in the radio-X-ray plane (van den Eijnden et al. 2020). However, recent observations of Swift J1858.6-0814 have shown qualitatively different X-ray properties, suggesting a state change while the source was unobservable due to Sun constraint (between 2019 November and 2020 February), although the properties of the initial phase were not typical of a canonical state (e.g. van der Klis 1994). In the 2020 observations, the flux level is much steadier and the strong iron line and edge are absent (Buisson et al. 2020b, and Figure 1), . These observations have also shown Type I X-ray bursts in both NICER and NUSTAR data (Buisson et al. 2020c), unambiguously identifying the compact object as a neutron star.

In this paper, we analyse the Type I X-ray bursts detected in NICER and NuSTAR data of Swift J1858.6-0814.

\section{OBSERVATIONS AND DATA REDUCTION}

We have inspected the NICER (Gendreau et al. 2016) light curves from 2020 by eye. Apparent Type I bursts are present in OBSIDs 3200400106, 3200400111, 3200400114, 3200400121 and 3200400122 , corresponding to March 6, 11, 14, 21 and 22 .

We begin with the calibrated, unfiltered events file from HEASARC (event_cl/ni32004001**_Ompu7_ufa.evt). We use the standard filters ${ }^{1}$ to produce good time intervals (GTIs) apart from the undershoot range, which we relax from $\leq 200 \mathrm{~s}^{-1}$ to $\leq 300 \mathrm{~s}^{-1}$ for the first Type I burst and $\leq 250 \mathrm{~s}^{-1}$ for the second. This is required due to high optieal loading due to the relatively low Sun angle. Additionally,

1 For further information on the filters, see heasarc.gsfc.nasa.gov/lheasoft/ftools/headas/nimaketime.html to include the peak of the second burst, we relax the offset from the nominal target direction slightly, using $0.0155^{\circ}$ rather than $0.015^{\circ}$. This is a small change from the standard value, so data during this time are unlikely to show significant deviations from the standard calibration. Further, the fourth burst occurs during passage through the South Atlantic Anomaly (SAA) and the overshoot rate reaches close to $5 \mathrm{~s}^{-1}$, so is removed by standard filtering. We remove these filters in order to show the light curve but note that the spectrum may be affected.

We then use NICERCLEAN to produce a clean events list, which we then barycentre to the ICRS reference frame and JPL-DE200 ephemeris. From this, we extract spectra and light curves using XSELECT.

We use NuSTAR (Harrison et al. 2013) OBSID 90601308002, which overlaps with NICER OBSID 3200400106. We reduce this using the standard NUPIPELINE and NUPRODUCTS software, version 1.9.0. We use a source region of a circle of radius 2 arcmin centred on the centroid of the detected counts. We use a background region of a circle of radius 2 arcmin from a source-free area of the detector.

\section{RESULTS AND DISCUSSION}

\subsection{Long-term light curve and burst recurrence time}

We show the light curve of Swift J1858.6-0814 since leaving Sun constraint on 2020 February 25 in Figure 1. The count rate shows a secular decrease throughout the whole of this period, punctuated by short dips and eclipses as well as the five Type I bursts analysed here. The drop in persistent count rate from the first to last burst was by a factor of around 4 and bursts were in general brighter at lower persistent count rate, with only the fourth burst not following this trend. Around the time of the last observed burst, the rate started decreasing more rapidly, before flattening once more. The period of fastest flux drop extended considerably before and after the final burst, so the coincidence in time is probably only by chance. As well as the Type I bursts, several dips are present, many of which are due to eclipses (Buisson et al. 2020c); these will be analysed in detail in future work.

The times between Type I bursts are 4.5, 3.6, 6.3 and 1.4 days (we summarise lists, such as this, of properties of each burst in Table A1). Since the coverage of Swift J1858.60814 is not continuous, there may have been other bursts between those observed, in observation gaps. Therefore, these gaps are an upper limit to the recurrence time. The duty cycle of NICER observations is low ( $\approx 3.9 \%$ over the 37 days shown in Figure 1, but not evenly across this time) so it is very likely that other bursts did occur outside times of observation. Furthermore, we can consider the $\alpha$-value, the ratio of inter-burst (persistent) fluence to burst fluence, which is typically $\approx 40$ for hydrogen fuelled bursts and $\approx 100-200$ for helium (e.g. Gottwald et al. 1986; Galloway et al. 2004). Here, the lowest observed $\alpha \approx 500$ (for burst 5 , integrating the fluxes found in Section 3.4) is higher, meaning more emission occurs between bursts than would be expected. This suggests that other, intermediate bursts did 
occur and/or substantial nuclear burning occurred between bursts.

There is also a period longer than any gap between observed bursts at the start of the NICER monitoring $(\approx 10$ days $)$ where no bursts are observed; again, it is possible that bursts did occur during this period but that they occurred during gaps in the NICER monitoring (which observed only 0.24 days of this time). The count rate and spectral shape show no large changes during this time, so there is no obvious reason for bursts not to have occurred. An alternative explanation for the lack of bursts in this period is that it followed a superburst, which quenched the normal Type I bursts (Keek et al. 2012); however, there is no evidence in the NICER monitoring or MAXI data (which cover earlier times) for a superburst having occurred.

\subsection{Confirmation of source of Type I bursts}

The first burst was observed by both NUSTAR and NICER. We show a NuSTAR image of the sky around Swift J1858.60814 in Figure 2. This shows that, to the resolution available to NUSTAR, only one source is apparent in the NICER field of view and the location of the Type I burst flux is consistent with the location of the persistent emission. The offset between the NuSTAR position and the nominal NICER pointing is around 15 arcsec, which is less than the 1 arcmin nominal pointing stability of NICER (Arzoumanian et al. 2014). This shows that the X-ray bursts are from Swift J1858.60814 .

\subsection{Type I X-ray burst light curves}

The light curves for each Type I burst are shown in Figure 3 . Each burst has a fast rise, lasting $\lesssim 3 \mathrm{~s}$, a single peak and fades to being undetectable over the persistent level within up to $\approx 40 \mathrm{~s}$. The decay of each burst, except the first, has an initial fast drop (within $\approx 2-3 \mathrm{~s}$ of the peak) followed by a slower exponential fade, lasting the remainder of the time (up to $\approx 40 \mathrm{~s}$ ) when the burst is observable over the persistent flux. This fast drop is by a greater factor in brighter bursts (Figure 4), for example, this drop is by a factor of $\approx 2.5$ in burst 1 but $\approx 4$ in burst 4. This shape is typical of Type I bursts fuelled by helium (Galloway et al. 2008a) Helium fuelled bursts can arise either when the accreted fuel is hydrogen poor or when accreted hydrogen burns stably between bursts; the binary orbital period is too long $(\approx 76840 \mathrm{~s}$, Buisson et al. 2020c) for a helium white dwarf companion and hydrogen is present in the optical spectra of the accretion disc/wind (Muñoz-Darias et al. 2020) so the latter case is more likely. The upper limits on the burst recurrence time (1.4 days in the best case) are long enough that sufficient hydrogen burning is plausible. The first burst is considerably fainter (peaking at $\approx 290 \mathrm{cts} / \mathrm{s}$ over $0.5-10 \mathrm{keV}$; the next faintest, burst 4 , peaks at $\approx 600 \mathrm{cts} / \mathrm{s}$ ) and shorter than the others. Apart from the fourth, each burst is stronger than the previous one, while the persistent count rate decreases; this could be due to partial burning of the accreted material occurring outside bursts producing more $\mathrm{H}$-poor fuel in the latter bursts, if more inter-burst burning occurred due to a longer recurrence time.

\subsection{Time resolved spectroscopy}

We extract time-resolved spectra for each Type I burst using time intervals containing a minimum number of photons. First, we estimate the persistent emission from the interval from $200 \mathrm{~s}$ to $50 \mathrm{~s}$ before the burst peak. We then define the start of the burst: we take a light curve binned to $0.1 \mathrm{~s}$ and find the final bin before the burst which is not above the persistent rate. We define the burst as starting at the end of this bin. Starting from this point, we extract spectra from time intervals containing at least 300 counts in excess of that expected from the persistent rate. We then fit the spectrum of the burst emission as the difference between each burst spectrum and the persistent spectrum (this is performed by treating the persistent emission as the background). We initially model the burst emission with a single blackbody. Apart from around the burst peaks, the spectra are described well by this model. However, spectra around the peaks of the second, third and fifth bursts are broader than a simple blackbody and in the fits show excess emission at low energies. We test two alternative phenomenological models to explain this excess: allowing the normalisation of the persistent emission to change by a factor $\left(1+f_{\mathrm{a}}\right)$ (Worpel et al. 2013) or adding a second blackbody. The former case requires a model for the persistent emission; we use TBABS $\times$ (DISKBB + BBODY), representing an absorbed disc and blackbody (we also use this model for the accretion rate estimates in Section 3.5). This soft state model gives a good fit to the persistent spectra for each burst (the worst case $\chi^{2} /$ d.o.f. $\left.=226.0 / 212=1.066, p=0.24\right)$ and agrees with various properties of the bursts (mentioned throughout this work) which match other bursts observed during the soft state

Both of these burst models provide good fits to all spectra (Figure 5) and provide similar peak fluxes and qualitative behaviour of the first blackbody component's radius around the burst peak. The total fit statistics for burst 5 for the spectra from times where a single black body gives a poor fit $\left(\chi_{\nu}^{2}>2\right)$ are: $\chi^{2} /$ d.o.f. $=78.7 / 76$ for the double black body model; and $\chi^{2} /$ d.o.f. $=73.5 / 83$ when varying the persistent emission. This implies a weak preference for a change in the strength of the persistent component but both models are statistically acceptable so we regard both options as possible. Parameters of the fits of each of these models to burst 5 are shown in Figure 6. Bursts 2 and 3 show similar features with lower signal; burst 1 has much lower signal; and we do not analyse burst 4 in detail due to the enhanced background from the SAA.

The area of the blackbody increases around the Type I burst peak before reducing and settling to a steady value for the majority of the burst tail, characteristic of photospheric radius expansion. Both well-fitting models (two blackbodies or additional persistent emission) show a similar degree of expansion, by around a factor of 2 over the radius in the tail of the burst, once the radius has settled to a steady value. The dip in blackbody radius after the burst peak to below the tail value is typical of bursts while accreting in the soft state, which agrees with our identification from the persistent spectrum, and is likely due to a changing colourcorrection factor (Güver et al. 2012a,b; Kajava et al. 2014).

These fits show a fast rise and smooth decay in bolometric flux. The apparent double peak in the flux curve for the 

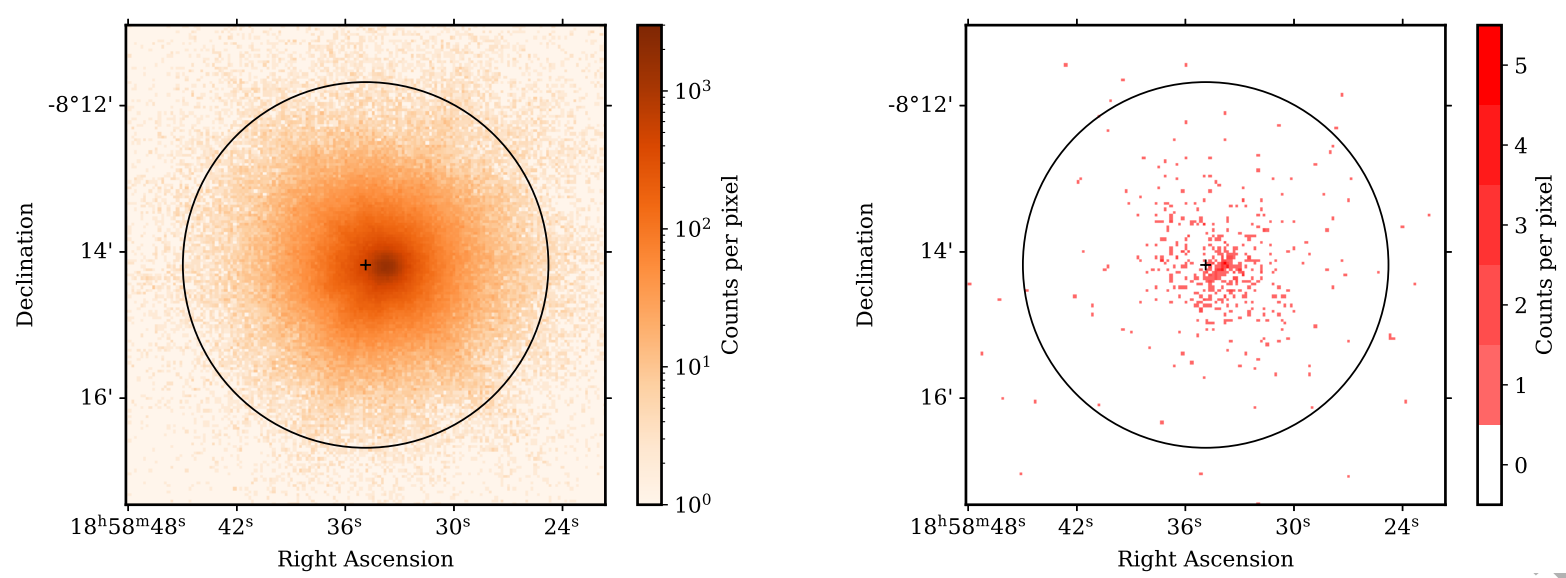

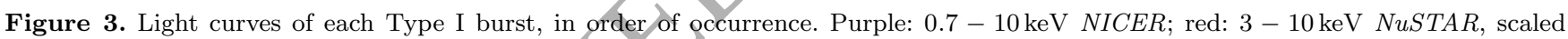
(increased by a factor of 5 ) and offset (by $+600 \mathrm{cts} \mathrm{s}^{-1}$ ). Each burst has had the persistent rate (the mean rate from $50-200 \mathrm{~s}$ before the burst) subtracted. The shaded regions are the $1 \sigma$ Poisson uncertainties.

single blackbody model is likely due to the poor fit around this time, although double peaks in bolometric luminosity have been seen in other PRE bursts (Jaisawal et al. 2019). The comparatively smooth flux profile contrasts with the fast drop in count rate after the peak; the difference being due to the higher temperatures early in the decay producing a lower count ratefor a given flux (when convolved with the instrument response, given the NICER effective area curve and the temperatures concerned).

Near the times of the Type I burst peaks (within about $2 \mathrm{~s}$ ), there is an excess of soft emission over the simple black body model. Similar excesses have been seen in Type I bursts in many other sources observed with NICER, e.g. Aql X1 (Keek et al. 2018a), 4U 1820-30 (Keek et al. 2018b) and SAX J1808.4-3658 (Bult et al. 2019). This could be due to other extra components such as re-emission from the disc (corresponding to the extra black body, Keek et al. 2018a) or enhanced accretion through Poynting-Robertson drag (corresponding to the change in persistent emission normalisation, Worpel et al. 2013). There may also be deviation from a simple blackbody due to Comptonisation (Keek et al. $2018 \mathrm{~b}$ ) or scattering processes in the atmosphere (Romani 1987). The data for the X-ray bursts presented here are not sensitive enough to distinguish between these possibilities clearly.

\subsection{Distance estimate and implications}

Since the later Type I bursts (certainly burst 5, with some evidence also in bursts 2 and 3) show photospheric radius expansion, their peak luminosity should be governed by the Eddington limit. The observed flux can then be used to estimate a distance. Initially, we use $L_{\mathrm{Edd}}=3.79 \times 10^{38} \mathrm{erg} / \mathrm{s}$, found empirically by Kuulkers et al. (2003) to be suitable 


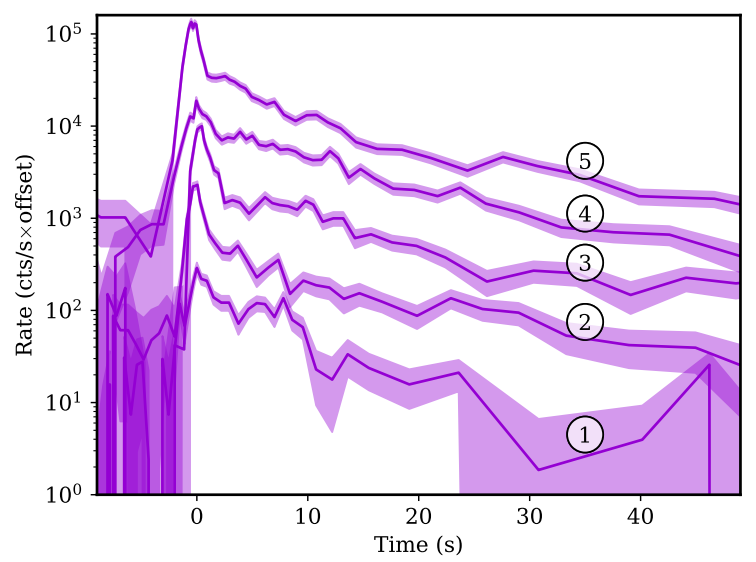

Figure 4. Light curves of each Type I burst, in order of occurrence from bottom to top. Purple: $0.7-10 \mathrm{keV}$ NICER. Each burst is offset from the previous by a factor of $10^{0.5}$. Each burst has had the persistent rate (the mean rate from $50-200 \mathrm{~s}$ before the burst) subtracted. The cooling tail is similar for each burst but bursts 2, 3 and 5 have a stronger initial peak. The shaded regions are the $1 \sigma$ Poisson uncertainties.

for neutron stars at known distance, and to have an accuracy of $15 \%$ for source-to-source variation. This matches the Eddington limit of a helium atmosphere around a $1.4 M_{\odot}$ object.

We take the peak flux from the second, third and fifth Type I bursts (which are consistent with each other; different temperatures mean that these correspond to different count rates). We use the model of the burst including a scaled persistent emission component (see Section 3.4), although the double black body model gives very similar results. For each burst, we use the least squares average of the fluxes from intervals which are consistent within $1-\sigma$ of the highest value. These values are consistent with each other and their average is $1.1 \pm 0.1 \times 10^{-8} \mathrm{erg} \mathrm{cm}^{-2} \mathrm{~s}^{-1}$, which gives a distance of

$$
D=16.7_{-1.3}^{+1.8} \mathrm{kpc}(1 \sigma)
$$

This puts Swift J1858.6-0814 at the far side of the Galaxy; given its Sky coordinates $(l=26.3894, b=$ -5.3237 ), this distance gives a Galactic (cylindrical) radius of $10 \mathrm{kpc}$ and a height of $1.5 \mathrm{kpc}$ below the Galactic plane.

Applying a prior for the relative density of the Galaxy along the line of sight/using the Galaxy model of Dehnen \& Binney 1998; Grimm et al. 2002; see also Gandhi et al. 2019) reduces this distance slightly, due to the higher density of objects, closer to the Galactic centre, giving

$$
D=16.2_{-1.6}^{+1.5} \operatorname{kpc}(1 \sigma) .
$$

From the relative densities of the components of the Galactic model at this position, we infer that Swift J1858.60814 is most likely $(75 \%)$ to be a disc object but could also be part of the halo (25\%). A bulge origin is highly unlikely $\left(P(\right.$ Bulge $\left.)=7 \times 10^{-6}\right)$.

There are systematic effects which may affect this dis-

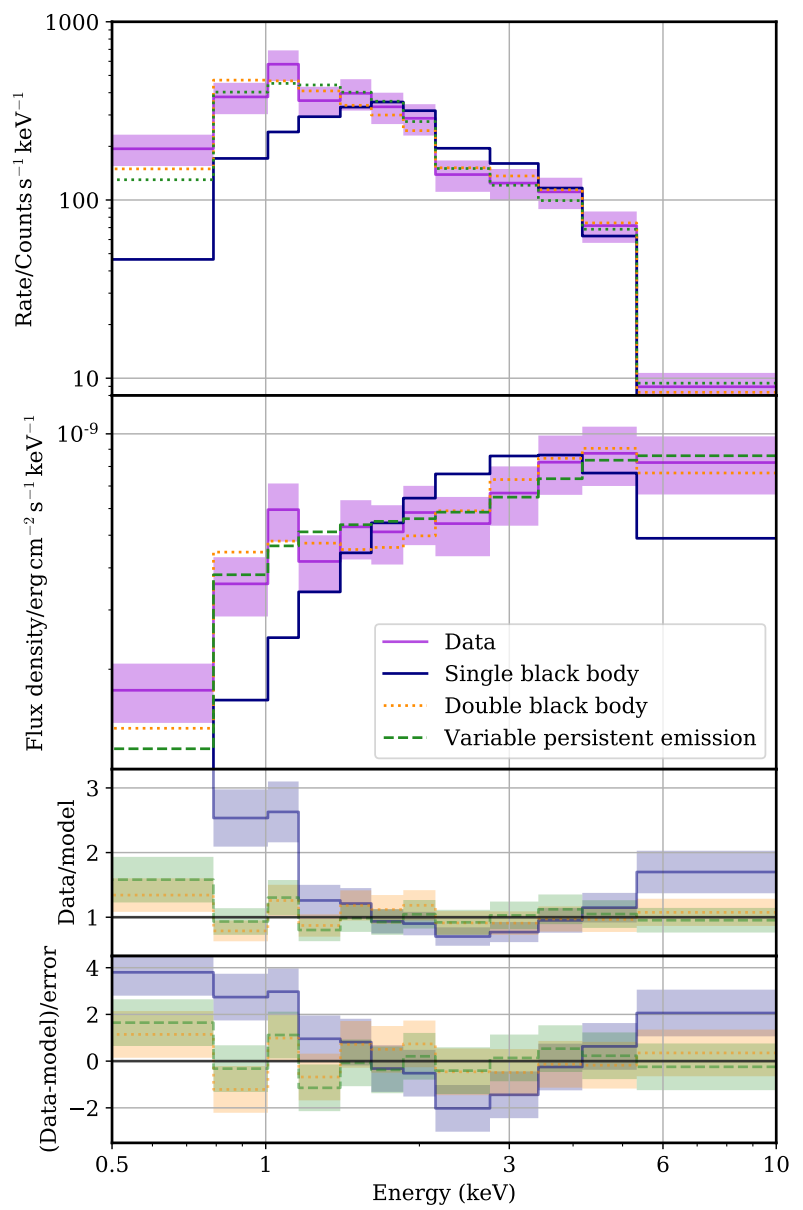

Figure 5. Comparison of different models for the net burst emission at the peak of the burst (the spectrum with highest count rate, in Burst 5). A single blackbody (navy) is a poor fit; two blackbodies (yellow) or a contribution proportional to the persistent flux (green) both give similarly good fits.

tance estimate (e.g. Galloway et al. 2008b). Many of these, such as differences in neutron star mass and photosphere metallicity, are implicitly included by the empirical nature of the critical luminosity (and its uncertainty) measured by Kuulkers et al. (2003). However, the effects of obscuration in high inclination sources are not accounted for Kuulkers et al. (2003) find that in some high inclination sources the observed PRE luminosity is significantly lower. In this case, the photosphere may be partially obscured by larger components of the system, principally the disc. For the simple case of a razor-thin disc, the disc can obscure up to half of the NS so the flux may be underestimated by up to a factor of 2 and the distance may actually be smaller by a factor of up to $\sqrt{2}$. This factor is mitigated by reflection of the radiation intercepted by the disc but may be increased by a thick disc He \& Keek (2016).

To show the magnitude of these effects, we show distance estimates for various specific values of metallicity and inclination in Figure 7 and Table 1. We calculate the distances by replacing the empirical peak luminosity from Kuulkers et al. (2003) with the theoretical Eddington luminosity (e.g. Lewin et al. 1993) modified by the anisotropy 


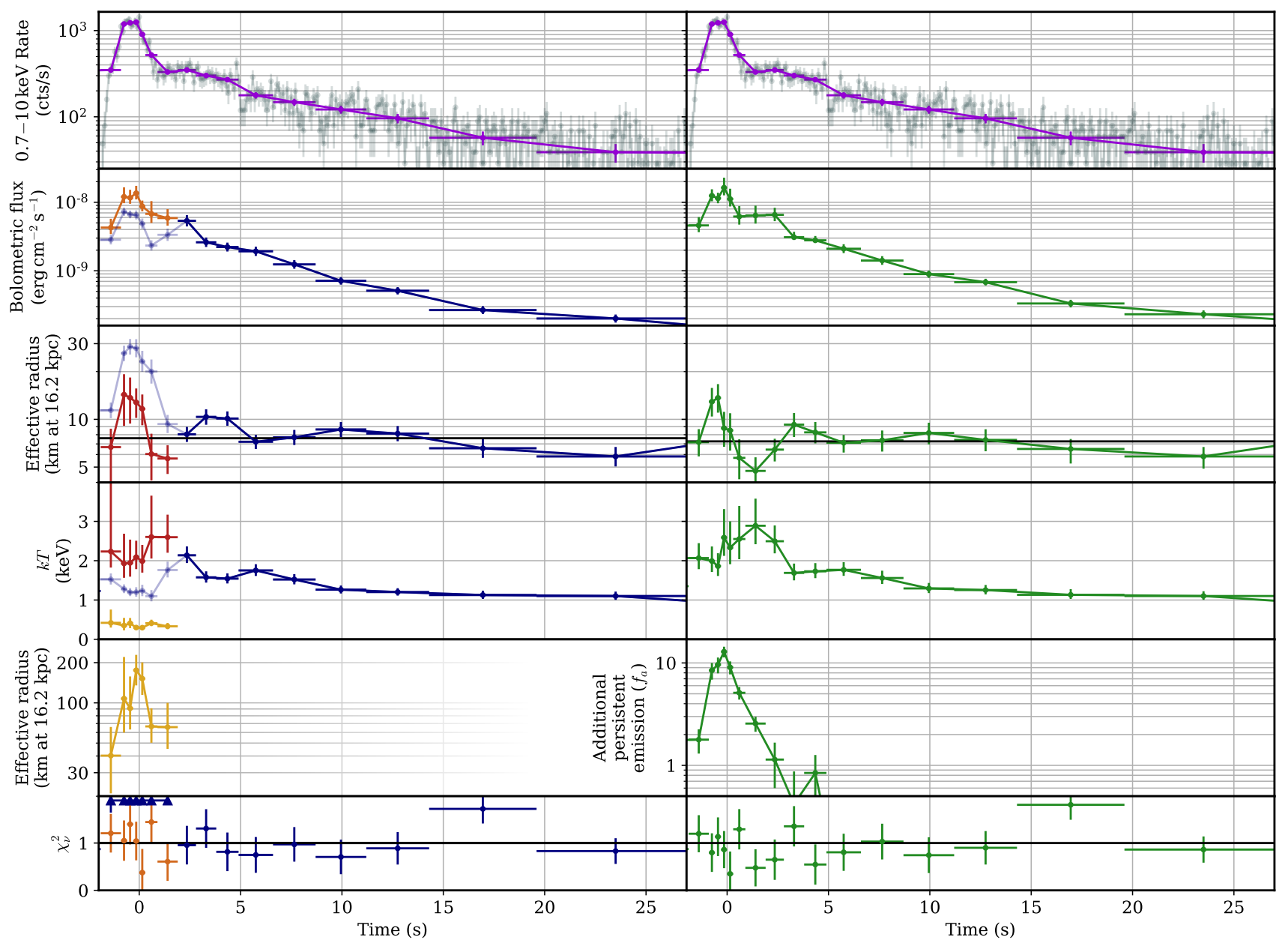

Figure 6. Parameters of time-resolved spectra of Burst 5. Left column: Parameters from modelling the Type I burst emission with 1 or 2 blackbodies. Parameters for a single blackbody model are/shown in navy; for two blackbodies, values for the complete model are shown in orange; the hotter blackbody in red and the cooler blackbody in yellow. The two-blackbody model is only shown where the single blackbody has $\chi_{\nu}^{2}>2$ and the single blackbody model is shown in faint navy in these cases. Right column: Parameters from modelling the burst with a blackbody and an increase in the normalisation of the persistent emission (green). Top panels: Lightcurves at $0.1 \mathrm{~s}$ resolution (grey) and binned to the times of the spectra (purple).

Table 1. Distance estimates (kpc) for various gas compositions and inclinations (see text for details).

\begin{tabular}{lccc}
\hline & Isotropic & $i=70^{\circ}$ & $i=80^{\circ}$ \\
\hline Pure helium & $16.6_{-0.8}^{+0.9}$ & $14.6_{-0.7}^{+0.8}$ & $12.8_{-0.6}^{+0.7}$ \\
Cosmic abundances & $12.1_{-0.6}^{+0.6}$ & $11.1_{-0.5}^{+0.6}$ & $9.8_{-0.4}^{+0.5}$ \\
\hline
\end{tabular}

factor $\left(\xi_{\mathrm{b}}\right)$ from (He \& Keek 2016),

$$
\widehat{L}_{\mathrm{O}, \mathrm{bs}} \Rightarrow \frac{8 \pi G m_{\mathrm{p}} M_{\mathrm{NS}} c}{\xi_{\mathrm{b}} \sigma_{\mathrm{T}}(1+X)(1+z(R))}
$$

where $G$ is the gravitational constant, $m_{\mathrm{p}}$ is the proton mass, $M_{\mathrm{NS}}$ is the neutron star mass, $c$ is the speed of light, $\sigma_{\mathrm{T}}$ is the Thomson cross section, $X$ is the hydrogen mass fraction and $z(R)$ is the gravitational redshift at the photospheric radius $R$. We show the two extremes of likely metallicity, pure helium $(X=0)$ and a cosmic abundance of hydrogen $(X=0.739)$. Since it shows eclipses (Buisson et al. 2020c),
Swift J1858.6-0814 is at high inclination; from He \& Keek (2016), the appropriate reduction in apparent luminosity $\left(\xi_{\mathrm{b}}^{-1}\right)$ for inclinations of $70-80^{\circ}$ is a factor of $0.85-0.65$. For each combination of parameters, we show the distance estimate for a neutron star mass of $1.4 M_{\odot}$ and a photospheric radius of $20 \mathrm{~km}$. This allows a significantly larger range of distances than the Kuulkers et al. (2003) range, due to the lower effective Eddington luminosities for high hydrogen fractions and high inclination. However, even the smallest distance estimate $\left(9.8_{-0.4}^{+0.5} \mathrm{kpc}\right)$ is $60 \%$ of the value derived from the Kuulkers et al. (2003) luminosity and beyond the average distance of Galactic sources along this line of sight.

To reduce the range of these estimates, we can consider whether particular values of parameters generating the systematic uncertainty are preferred by other evidence. The eclipse duration implies an inclination of at least $70^{\circ}$ (Buisson et al. 2020c, Buisson et al. in prep.). A more accurate determination of the inclination would require detailed 


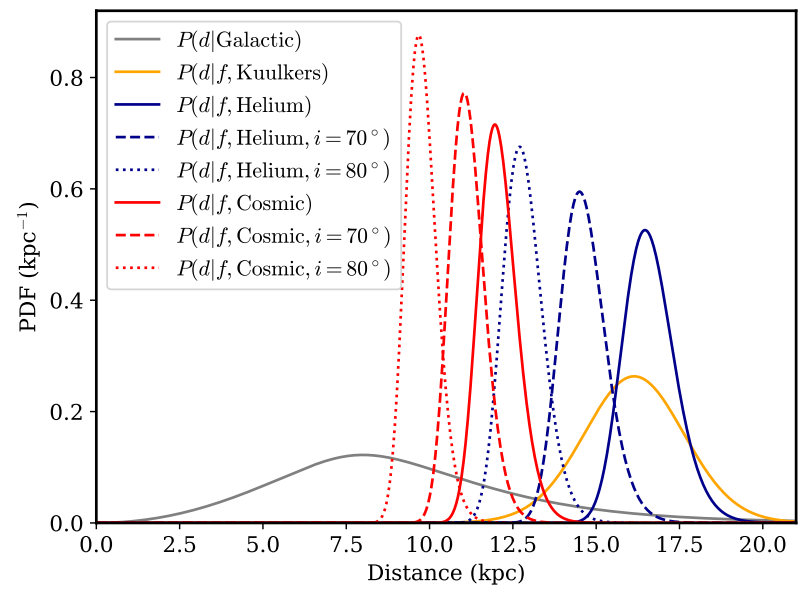

Figure 7. Distance estimates for Swift J1858.6-0814 based on various critical luminosities for PRE. Distance estimates for several specific luminosity values are shown (details in legend and text) along with the empirical luminosity range found by Kuulkers et al. (2003). The Galactic prior is shown in grey.

modelling of optical light curves and spectra, beyond the scope of this paper; meanwhile, we regard our calculation using $80^{\circ}$ as a fiducial value. The atmospheric composition of a Type I burst can be inferred from its light curve. The relatively fast rise and initial decay of the PRE bursts observed here suggest a helium burst. Additionally, helium fuelled bursts are also more common during the soft accretion state and the dip in apparent radius below the final value is more typical of soft state bursts (Kajava et al. 2014). Further, bursts can reach the Eddington limit for helium even where accreted material is hydrogen rich, either by the hydrogen being burnt between bursts or the hydrogen rich atmosphere being blown off by the burst (Bult et al. 2019; Galloway et al. 2006). This would imply that the further distance estimates (blue curves in Figure 7) are more likely $\left(12.8_{-0.6}^{+0.7} \mathrm{kpc}\right.$ for $\left.i=80^{\circ}\right)$.

With this distance estimate, we can also estimate the accretion rate at the times of the bursts from the persistent flux measurements from the modelling in Section 3.5. We find a bolometric (of the X-ray components) flux before the bursts of $12.6_{-0.4}^{40.5}, 10.2_{-0.4}^{+0.5}, 8.9_{-0.4}^{+0.6}$, $5.6_{-0.3}^{+0.5}$ and $2.3_{-0.15}^{+0.2} \times 10^{-10} \mathrm{erg} \mathrm{cm}^{-2} \mathrm{~s}^{-1}$, in chronological order. If the persistent emission has the same anisotropy as the burst, this implies an Eddington fraction $\dot{m}_{\text {Edd }}=$ $0.20_{-0.02}^{+0.01}, 0.16_{-0.02}^{+0.01}, 0.14_{-0.02}^{+0.01}, 0.09_{-0.01}^{+0.01}$ and $0.036 \pm 0.004$ for material in the accretion flow (calculating $L_{\mathrm{Edd}}$ for $X=$ 0.73 ). However, the disc and boundary layer may have more anisotropic emission than the burst from the NS surface (e.g. He \& Keek 2016), so the true Eddington fraction could be somewhat higher. The exact factor depends on the details of the accretion structure and the inclination; for a flat disc (which provides all the persistent flux) observed at $70-80^{\circ}$, the increase is by a factor of 1.2-2. This is similar to the range at which helium fuelled bursts are expected and observed (Galloway et al. 2008a) but extends slightly higher, so there could be some influence of residual hydrogen in the burning material.

Our distance estimates are all relatively large
(Galloway et al. 2008a; Gandhi et al. 2019) but not unprecedented (e.g. Homan et al. 2014) for an XRB. The absorbing column density $\left(\approx 2 \times 10^{21} \mathrm{~cm}^{-2}\right)$ is comparatively low for such a distant source, but the total Galactic column density in the direction of Swift J1858.6-0814 is similar $\left(1.8 \times 10^{21} \mathrm{~cm}^{-2}\right.$, HI4PI Collaboration et al. 2016) .

A large distance can also help in explaining the strong variability observed in the initial state of Swift J1858.60814 (during 2018-9): it is comparatively faint for a binary but strong winds (Muñoz-Darias et al. 2020) and variability (Ludlam et al. 2018) are often explained by a high Eddington rate (King \& Pounds 2003; Grupe 2004). During the flaring state but between flares, the observed flux of Swift J1858.6-0814 was $\approx 2.5 \times 10^{-10} \mathrm{erg} \mathrm{cm}^{-2} \mathrm{~s}^{-1}$ (Hare et al. 2020) $)^{2}$, which is $\approx 5 \%$ of the Eddington limit for hydrogen and a $1.4 M_{\odot}$ object. Some flares increased count rates by factors of many tens, so at least during bright flares, the luminosity was above the Eddington luminosity/and correcting for any anisotropy is likely only to increase the strength of this). If much of the variability was due to obscuration, the intrinsic luminosity would also have been above Eddington at other times.

\subsection{Pre-burst oscillations}

We also looked for millihertz quasi-periodic oscillations ( $\mathrm{mHz}$ QPOs), which are sometimes found before an X-ray burst (e.g. Revnivtsev etal. 2001; Altamirano et al. 2008; Mancuso et al. 2019). We used $0.5-10 \mathrm{keV}$ light curves at 1 second resolution and applied the Lomb-Scargle periodogram (Lomb 1976; Scargle 1982) to each gap-less light curve, excluding periods of dipping and eclipses. In the five cases where we detected the type-I X-ray bursts, we searched for the oscillations before and after the X-ray bursts. To estimate the significance level, we followed the approach of (Press et al. 1992), which assumes white noise and takes as a number of trials the number of frequencies explored.

We detected a mHz QPO at a significance of $5.8 \sigma$ in the $1.8 \mathrm{ks}$ of data before the 5 th X-ray burst (Figure 8 ). The $\mathrm{mHz} \mathrm{QPO}$ has an average frequency of $9.6 \pm 0.5 \mathrm{mHz}$ and a fractional $\mathrm{rms}$ amplitude of $2.2 \pm 0.2 \%(0.5-10 \mathrm{keV})$. There is no evidence of the oscillations in the $\approx 600 \mathrm{~s}$ of data after the X-ray burst, with a $90 \%$ upper limit on the rms amplitude of $1.2 \%$ ruling out that the same strength of oscillation continues. We also found marginal evidence of QPOs in at least three other cases; however the datasets are relatively short $(\lesssim 500-700 \mathrm{~s})$, and therefore it is not possible to understand if they are real or the product of red-noise. The upper limits to the QPO amplitude for the time segments prior to the earlier bursts are somewhat lower in fractional amplitude (0.6, 0.8, 1.0 and $0.8 \%$ in chronological order) than for the detected QPO but due to the brighter flux at earlier times, all but the last of these limits are higher in absolute amplitude than the detected QPO. Therefore, we cannot definitively rule out a QPO from the same level of oscillatory burning occurring prior to the other bursts. We also

2 This was measured for the $3-78 \mathrm{keV}$ band, which for the spectral shape of this observation includes the majority of flux; any bolometric correction will only increase the strength of superEddington behaviour. 

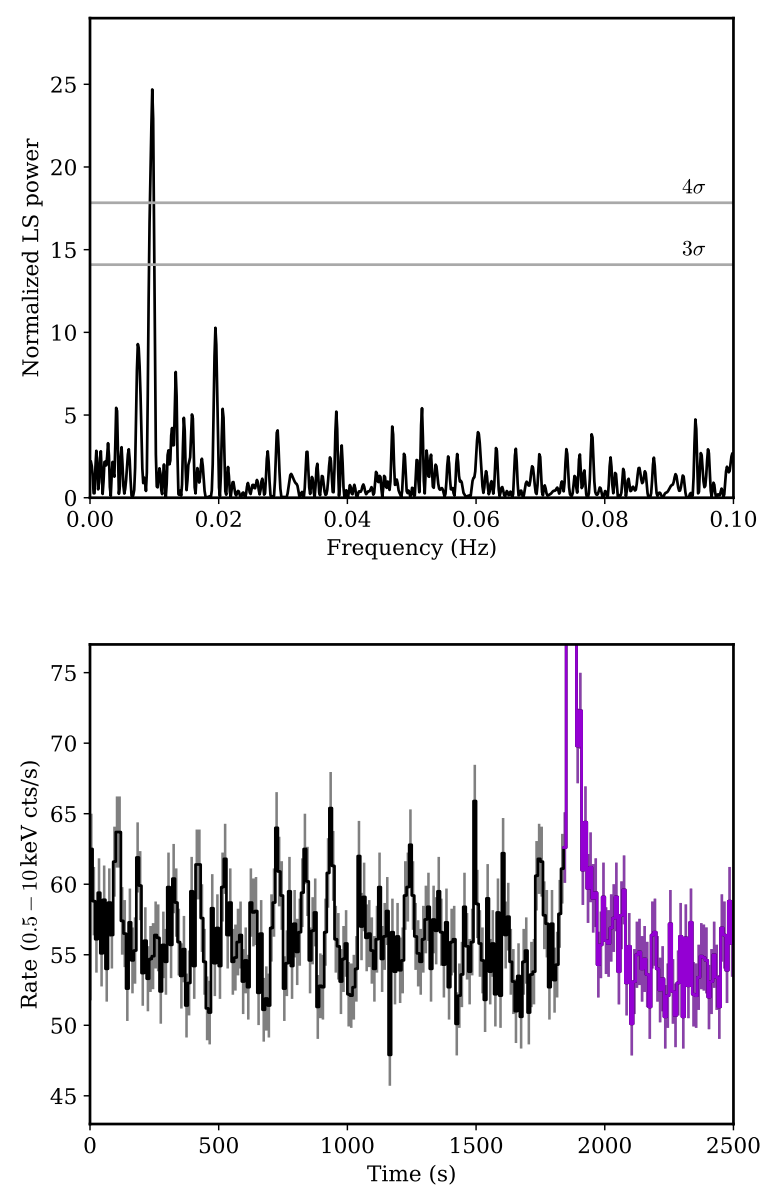

Figure 8. Top: Lomb-Scargle periodogram of the light curve segment immediately prior to burst 5 , which shows a QPO at $9.6 \pm 0.5 \mathrm{mHz}$. Bottom: $0.5-10 \mathrm{keV}$ NICER light curve of the period up to and including burst 5 , during which a $9.6 \pm 0.5 \mathrm{mHz}$ QPO is detected. The burst itself (purple) far exceeds the plotted range.

note that a $\mathrm{mHz}$ QPO was also detected in a NuSTAR observation during 2019 February (Hare et al. 2019), although at a frequency of $2.7 \mathrm{mHz}$, which is lower than other $\mathrm{mHz}$ QPOs which have been explained by marginally stable nuclear burning.

Revnivtsev et al. (2001) find that these $\mathrm{mHz}$ QPOs are only found in a narrow range of luminosities, $L_{3-20 \mathrm{keV}}=$ $5-11 \times 10^{36} \mathrm{erg} \mathrm{s}^{-1}$. The QPO found here occurs while the mean flux (from our model for the persistent emission, Section 3.4) is $f_{3-20 \mathrm{keV}}=1.4 \pm 0.2 \times 10^{10} \mathrm{erg} \mathrm{cm}^{-2} \mathrm{~s}^{-1}$, which corresponds to $L_{3}-20 \mathrm{keV}=4.3_{-0.5}^{+0.6} \times 10^{36} \mathrm{erg} \mathrm{s}^{-1}$ at $16.2 \mathrm{kpc}$ (derived from the Type I burst peak luminosity for the population, since we are comparing with a population luminosity for other QPO detections), which supports the upper end of the distance estimates found here. Using a lower distance to account for the effect of high inclination in Swift J1858.60814 would imply a lower luminosity, which is compatible with the luminosities at which QPOs are observed in other sources if this also depends on inclination, with at least as strong an anisotropy factor as the burst emission. This would be expected for disc, NS surface or coronal emission so long as this is not strongly equatorially beamed.

The characteristics of the $\mathrm{mHz}$ QPOs we found here are consistent with those found in 6 other NS systems (Revnivtsev et al. 2001; Altamirano et al. 2008; Strohmayer \& Smith 2011; Lyu et al. 2014, 2015; Strohmayer et al. 2018; Mancuso et al. 2019, but compare Linares et al. 2012) and are usually explained as being the product of marginally stable burning of He on the NS surface (Heger et al. 2007). This is the $7^{\text {th }}$ NS system that shows this type of QPOs. The fact that we do not detect more episodes of $\mathrm{mHz}$ QPOs could either be due to their intrinsic absence or to detection difficulty: the $\mathrm{mHz}$ QPOs are not always present in the X-ray light curves (they are state dependent and even in a given state, there is not yet a clear physical trigger for them, see Altamirano et al. 2008; Mancuso et al. 2019 , etc.); in addition, the frequency and amplitude of these QPOs are very low, and therefore to acquire enough QPO cycles and sufficient signal-to-noise, uninterrupted datasets longer than $1000-1500 \mathrm{~s}$ are generally needed to unambiguously detect them.

\subsection{Burst oscillation search}

We searched each of the X-ray bursts observed with NICER for the presence of burst oscillations, but did not detect any significant signals. To search for oscillations, we constructed a 1/8192 s time resolution light curve for each X-ray burst, using only those events in the $1-8 \mathrm{keV}$ energy band. These light curves all started 10 seconds prior to the burst onset, and had durations of 40 seconds. For each considered X-ray burst, we applied a $T=2,4,8 \mathrm{~s}$ duration window selection, which we moved across the burst profile in steps of $T / 2$. We then calculated the power spectrum associated with each window position, and searched the $100-2000 \mathrm{~Hz}$ frequency range for excess power over the expected noise distribution. No such excess was observed, to a $95 \%$ confidence upper limit of approximately $15 \%$ fractional amplitude in the most sensitive segment (the peak light curve of burst $5)$. We note, however, that the vast majority of considered segments had much lower averaged count-rates, and thus substantially higher upper-limits. With typical upper limits ranging between $30 \%$ and $80 \%$ fractional amplitude, our results are therefore not especially constraining.

\section{FURTHER DISCUSSION}

\subsection{Implications of the neutron star accretor}

The identification of the accretor in Swift J1858.6-0814 as a neutron star informs several outstanding questions relating to the properies of Swift J1858.6-0814. It fits with the low coronal temperature found in Hare et al. (2020), since neutron stars tend to have lower coronal temperatures than black holes (Burke et al. 2017).

However, the neutron star accretor implies an unusual location in the radio-X-ray plane: Swift J1858.60814 appears relatively X-ray faint for a NS XRB (van den Eijnden et al. 2020). This could imply that Swift J1858.6-0814 has an intrinsically unusually low Xray/radio luminosity ratio or that the observed X-ray lu- 
minosity is unrepresentatively low. The latter case would support a model in which the X-ray emission (which may already be comparatively low due to anisotropy, e..g. He \& Keek 2016) is usually obscured by the high inclination disc, apart from during the flares, which represent the true intrinsic luminosity, when viewing the central source directly through a gap in the (irregular) disc surface.

Swift J1858.6-0814 has previously been compared with the black hole XRBs V4641 Sgr and V404 Cyg (e.g. Hare et al. 2020). All of these sources have shown strong variability due to some combination of changes in intrinsic flux and obscuration, although the relative contribution of these two effects is not yet clear (e.g. compare Walton et al. 2017; Koljonen \& Tomsick 2020). The relative radio loudness also provides a further similarity with V404 Cyg, which is unusually radio loud for its inclination (Motta et al. 2018). The identification of Swift J1858.6-0814 as a neutron star $\mathrm{XRB}$ means the flaring behaviour in these sources must now be explained in a model which is compatible with a neutron star accretor. In particular, extreme variability from processes very close to the event horizon may be ruled out, since a neutron star is significantly larger than its Schwarzschild radius.

\subsection{Bursts in the flaring state?}

Swift J1858.6-0814 had been active for over a year before any Type I bursts were detected; there are several means to explain the non-detection of bursts during this period. Firstly, there may truly have been no bursts, due to the different accretion regime during this period. In a model where variable obscuration causes much of the strong variability, the intrinsic accretion rate was much higher during the flaring period, so would likely have induced stable nuclear burning of both hydrogen and helium. Additionally, in this model, the obscuration between flares would have impeded observation of any Type I bursts which occurred while the neutron star was obscured (which is the majority of the duty cycle). It is also possible that bursts were observed but not identified if they occurred at the same time as flares. The observed flares are all different in spectrum, light curve and/or duration to thermonuclear bursts; however, the variety of flares means that it is possible that a burst coincident with a flare would go unnoticed. Finally, it is also possible that bursts did occur during this phase of the outburst but, by chance, not during NICER observations of Swift J1858.6-0814. Overall, it is unsurprising that X-ray bursts had not been detected in the flaring state, whether or not they occurred.

\subsection{Comparison with other similar sources}

We can also compare the flaring state to other strong variability regimes in neutron stars. Two famous neutron star systems exhibiting flare-like behaviour are the Rapid Burster (MXB 1730-335, e.g. Hoffman et al. 1978) and Bursting Pulsar (GRO J1744-28, Fishman et al. 1995). The Rapid Burster shows many (up to thousands per day) 'rapid' bursts in addition to Type I bursts; these rapid bursts are much shorter $(<10 \mathrm{~s})$ and more regular in cadence than the flares of Swift J1858.6-0814, so are probably different phenomena.
The Bursting Pulsar is the archetypal example of Type II Xray bursts (Kouveliotou et al. 1996). These bursts also differ markedly from the flares observed in Swift J1858.6-0814: the type II bursts are again much shorter and are accompanied by a drop in emission following the burst. Therefore, the flaring state of Swift J1858.6-0814 is not explained as an example of these other unusual neutron star XRB states.

The high inclination NS LMXB EXO 0748-676 has also shown flaring episodes (Homan et al. 2003), although these are more sporadically interspersed with other light curve shapes and less prominent at harder energies than those in Swift J1858.6-0814.

Transitional millisecond pulsars (tMSPs) also have a 'flaring' accretion mode (de Martino et al. 2013; Bogdanov \& Halpern 2015), although this occurs at much lower luminosity $\left(\approx 10^{34} \mathrm{erg} \mathrm{s}^{-1}\right)$ than the flaring state in Swift J1858.6-0814 ( The tMSP flaring mode can also show strong, variable absorption (e.g. Li et al. 2020), so could be an analogue with lower accretion efficiency.

There have not yet been measurements of the magnetic field strength in Swift J1858.6-0814; the closer comparison of the flaring state of Swift J1858.6-0814 with black hole than neutron star systems could be because the magnetic field of its neutron star is low enough to be unimportant in its accretion flow, implying a relatively low magnetic field strength.

\section{ACKNOWLEDGEMENTS}

We thank Laurens Keek for helpful discussions and the referee for comments which improved the manuscript. We thank the NuSTAR operations team for rapid approval and execution of our Target of Opportunity proposal. D.J.K.B. and D.A. are funded by the Royal Society. T.G. has been supported in part by the Scientific and Technological Research Council (TÜBITAK) 119F082, Royal Society Newton Advanced Fellowship, NAF $\backslash$ R2 $\backslash 180592$, and Turkish Republic, Directorate of Presidential Strategy and Budget project, 2016K121370. J. Hare acknowledges support from an appointment to the NASA Postdoctoral Program at the Goddard Space Flight Center, administered by the Universities Space Research Association under contract with NASA. C.M. is supported by an appointment to the NASA Postdoctoral Program at the Marshall Space Flight Center, administered by the Universities Space Research Association under contract with NASA. M.O.A. acknowledges support from the Royal Society through the Newton International Fellowship programme. This work made use of data from the NuSTAR mission, a project led by the California Institute of Technology, managed by the Jet Propulsion Laboratory, and funded by the National Aeronautics and Space Administration. This research has made use of the $N u S$ $T A R$ Data Analysis Software (NuSTARDAS) jointly developed by the ASI Science Data Center (ASDC, Italy) and the California Institute of Technology (USA). NICER is a mission of NASA's Astrophysics Explorers Program. This research has made use of data and software provided by the High Energy Astrophysics Science Archive Research Center (HEASARC), which is a service of the Astrophysics Science Division at NASA/GSFC and the High Energy Astro- 
physics Division of the Smithsonian Astrophysical Observatory. This research has made use of ISIS functions (ISISscripts) provided by ECAP/Remeis observatory and MIT (http://www.sternwarte.uni-erlangen.de/isis/).

\section{DATA AVAILABILITY}

The data underlying this article are available in HEASARC.

\section{REFERENCES}

Altamirano D., van der Klis M., Wijnands R., Cumming A., 2008, ApJ, 673, L35

Arzoumanian Z., et al., 2014, The neutron star interior composition explorer (NICER): mission definition. p. 914420, doi:10.1117/12.2056811

Bildsten L., 1995, ApJ, 438, 852

Bildsten L., 1998, in Buccheri R., van Paradijs J., Alpar A., eds, NATO Advanced Science Institutes (ASI) Series C Vol. 515, NATO Advanced Science Institutes (ASI) Series C. p. 419 (arXiv: astro-ph/9709094)

Bogdanov S., Halpern J. P., 2015, ApJ, 803, L27

Bolton C. T., 1972, Nature Physical Science, 240, 124

Brown E. F., Bildsten L., Rutledge R. E., 1998, ApJ, 504, L95

Buisson D. J. K., et al., 2020a, MNRAS,

Buisson D. J. K., Altamirano D., Remillard R., Arzoumanian Z., Gendreau K., Gandhi P., Vincentelli F., 2020b, The Astronomer's Telegram, 13536, 1

Buisson D. J. K., et al., 2020c, The Astronomer's Telegram, 13563, 1

Bult P., et al., 2019, ApJ, 885, L1

Burke M. J., Gilfanov M., Sunyaev R., 2017, MNRAS, 466, 194

Casares J., Charles P. A., Naylor T., 1992, Nature, 355, 614

Cornelisse R., Heise J., Kuulkers E., Verbunt F., in't Zand J. J. M., 2000, A\&A, 357, L21

Cumming A., Bildsten L., 2001, ApJ, 559, L127

Cumming A., Macbeth J., 2004, ApJ, 603, L37

Dehnen W., Binney J., 1998, MNRAS, 294, 429

Fishman G. J., Kouveliotou C., van Paradijs J., Harmon B. A., Paciesas W. S., Briggs M. S., Kommers J., Lewin W. H. G., 1995, IAU Circ., 6272, 1

Fujimoto M. Y., Hanawa T., Miyaji S., 1981, ApJ, 247, 267

Gallo E., Degenaar N., van den Eijnden J., 2018, MARAS,

478, L132
Galloway D. K., Keek L., 2017, arXiv e-prints, p. arXiv: 1712.06227

Galloway D. K., Cumming A., Kuulkers E., Bildsten L., Chakrabarty D., Rothschild R. E., 2004, ApJ, 601, 466

Galloway D. K., Psaltis D., Muno M. P., Chakrabarty D., 2006, ApJ, 639, 1033

Galloway D. K., Muno M. P., Hartman J. M., Psaltis D., Chakrabarty D., 2008a, ApJS, 179, 360

Galloway D. K., Özel F., Psaltis D., 2008b, MNRAS, 387, 268

Galloway D. K., et al., 2018, ApJ, 857, L24

Gandhi P., et al., 2016, MNRAS, 459, 554

Gandhi P., Rao A., Johnson M. A. C., Paice J. A., Maccarone T. J., 2019, MNRAS, 485, 2642

Gendreau K. C., et al., 2016, in Space Telescopes and Instrumentation 2016: Ultraviolet to Gamma Ray. p. 99051H, doi: 10.1117/12.2231304

GottwaldM., Haberl F., Parmar A. N., White N. E., 1986, ApJ, 308,213

Grimm H. J., Gilfanov M., Sunyaev R., 2002, A\&A, 391, 923

Grindlay J., Gursky H., Schnopper H., Parsignault D. R., Heise J., Brinkman A. C., Schrijver J., 1976, ApJ, 205, L127
Grupe D., 2004, AJ, 127, 1799

Güver T., Psaltis D., Özel F., 2012a, ApJ, 747, 76

Güver T., Özel F., Psaltis D., 2012b, ApJ, 747, 77

HI4PI Collaboration et al., 2016, A\&A, 594, A116

Hare J., Gandhi P., Paice J. A., Tomsick J., 2019, The Astronomer's Telegram, 12512, 1

Hare J., et al., 2020, arXiv e-prints, p. arXiv:2001.03214

Harrison F. A., et al., 2013, ApJ, 770, 103

He C. C., Keek L., 2016, ApJ, 819, 47

Heger A., Cumming A., Woosley S. E., 2007, ApJ, 665, 1311

Hewish A., Bell S. J., Pilkington J. D. H., Scott P. F., Collins R. A., 1968, Nature, 217, 709

Hoffman J. A., Marshall H. L., Lewin W. H. G., 1978, Nature, 271,630

Homan J., Wijnands R., van den Berg M., 2003, A\&A, 412, 799

Homan J., Fridriksson J. K., Wijnands R., Cackett E. M., Degenaar N., Linares M., Lin D., Remillard R. A., 2014, ApJ, 795,131

Jaisawal G. K., et al., 2019, ApJ, 883, 61

Kajava J. J. E., et al., 2014, MNRAS, 445, 4218

Keek L., Heger A., in't Zand J. J. M., 2012, ApJ, 752, 150

Keek L., et al., 2018a, ApJ, 855, L4

Keek L., et al., 2018b, ApJ, 856, L37

Kennea J. A., Krimm H. A., 2018, The-Astronomer's Telegram, 12160, 1

King A. R., Pounds K. A., 2003, MNRAS, 345, 657

Koljonen K. I. I., Tomsick J. A., 2020, arXiv e-prints, p. arXiv:2004.08536

Kouveliotou C., van Paradijs J., Fishman G. J., Briggs M. S., Kommers J., Harmon B. A., Meegan C. A., Lewin W. H. G., 1996, Nature, 379, 799

Krimm H. A., et al., 2018, The Astronomer's Telegram, 12151, 1

Kuulkers E., den Hartog P. R., in't Zand J. J. M., Verbunt F. W. M., Harris W. E., Cocchi M., 2003, A\&A, 399, 663

Lewin W. H. G., Vacca W. D., Basinska E. M., 1984, ApJ, 277, L57

Lewin W. H. G., van Paradijs J., Taam R. E., 1993, Space Sci. Rev., 62, 223

Li K.-L., Strader J., Miller-Jones J. C. A., Heinke C. O., Chomiuk L., 2020, arXiv e-prints, p. arXiv:2004.14573

Linares M., Altamirano D., Chakrabarty D., Cumming A., Keek L., 2012, ApJ, 748, 82

Lomb N. R., 1976, Ap\&SS, 39, 447

Ludlam R. M., et al., 2018, The Astronomer's Telegram, 12158, 1

Lyu M., Méndez M., Altamirano D., 2014, MNRAS, 445, 3659

Lyu M., Méndez M., Zhang G., Keek L., 2015, MNRAS, 454, 541

Lyu M., Méndez M., Altamirano D., Zhang G., 2016, MNRAS, 463,2358

Mancuso G. C., Altamirano D., García F., Lyu M., Méndez M., Combi J. A., Díaz-Trigo M., in’t Zand J. J. M., 2019, MNRAS, 486, L74

McClintock J. E., Remillard R. A., 2006, Black hole binaries. pp 157-213

Migliari S., Fender R. P., 2006, MNRAS, 366, 79

Motta S. E., Kajava J. J. E., Sánchez-Fernández C., Giustini M., Kuulkers E., 2017, MNRAS, 468, 981

Motta S. E., Casella P., Fender R. P., 2018, MNRAS, 478, 5159

Muñoz-Darias T., et al., 2020, arXiv e-prints, p. arXiv:2003.12073

Munoz-Darias T., Jimenez-Ibarra F., Armas Padilla M., Casares J., Cuneo V., Panizo-Espinar G., Sanchez-Sierras J., Torres M. A. P., 2019, The Astronomer's Telegram, 12881, 1

Narayan R., Heyl J. S., 2003, ApJ, 599, 419

Orosz J. A., Bailyn C. D., 1997, ApJ, 477, 876

Orosz J. A., et al., 2001, ApJ, 555, 489

Paice J. A., Gandhi P., Dhillon V. S., Marsh T. R., Green M., Breedt E., 2018, The Astronomer's Telegram, 12197, 1

Patruno A., Watts A. L., 2012, arXiv e-prints, p. arXiv:1206.2727 
Press W. H., Teukolsky S. A., Vetterling W. T., Flannery B. P., 1992, Numerical recipes in FORTRAN. The art of scientific computing

Revnivtsev M., Churazov E., Gilfanov M., Sunyaev R., 2001, A\&A, 372, 138

Revnivtsev M., Gilfanov M., Churazov E., Sunyaev R., 2002, A\&A, 391, 1013

Reynolds M. T., Miller J. M., Ludlam R. M., Tetarenko B. E., 2018, The Astronomer's Telegram, 12220, 1

Romani R. W., 1987, ApJ, 313, 718

Scargle J. D., 1982, ApJ, 263, 835

Spitkovsky A., Levin Y., Ushomirsky G., 2002, ApJ, 566, 1018

Strohmayer T., Bildsten L., 2006, New views of thermonuclear bursts. pp 113-156

Strohmayer T. E., Brown E. F., 2002, ApJ, 566, 1045

Strohmayer T. E., Smith E. A., 2011, The Astronomer's Telegram, 3258, 1

Strohmayer T. E., Zhang W., Swank J. H., Smale A., Titarchuk L., Day C., Lee U., 1996, ApJ, 469, L9

Strohmayer T. E., et al., 2018, ApJ, 865, 63

Tawara Y., et al., 1984, ApJ, 276, L41

Walton D. J., et al., 2017, ApJ, 839, 110

Webster B. L., Murdin P., 1972, Nature, 235, 37

Wijnands R., van der Klis M., 2000, ApJ, 528, L93

Worpel H., Galloway D. K., Price D. J., 2013, ApJ, 772, 94

Zdziarski A. A., Lubiński P., Smith D. A., 1999, MNRAS, 303, L11

de Martino D., et al., 2013, A\&A, 550, A89

in't Zand J., 2017, in Serino M., Shidatsu M., Iwakiri W., Mihara T., eds, 7 years of MAXI: monitoring X-ray Transients. p. 121 (arXiv: 1702.04899)

van Paradijs J., 1978, Nature, 274, 650

van den Eijnden J., et al., 2020, MNRAS, 496, 4127

van der Klis M., 1994, ApJS, 92, 511

van der Klis M., 2006, Rapid X-ray Variability. pp 39-112

van der Klis M., Swank J. H., Zhang W., Jahoda K., Morgan E. H., Lewin W. H. G., Vaughan B., van Paradijs J., 1996, ApJ, 469, L1

\section{APPENDIX A: SUMMARY OF BURST PROPERTIES}


Table A1. Summary of burst properties.

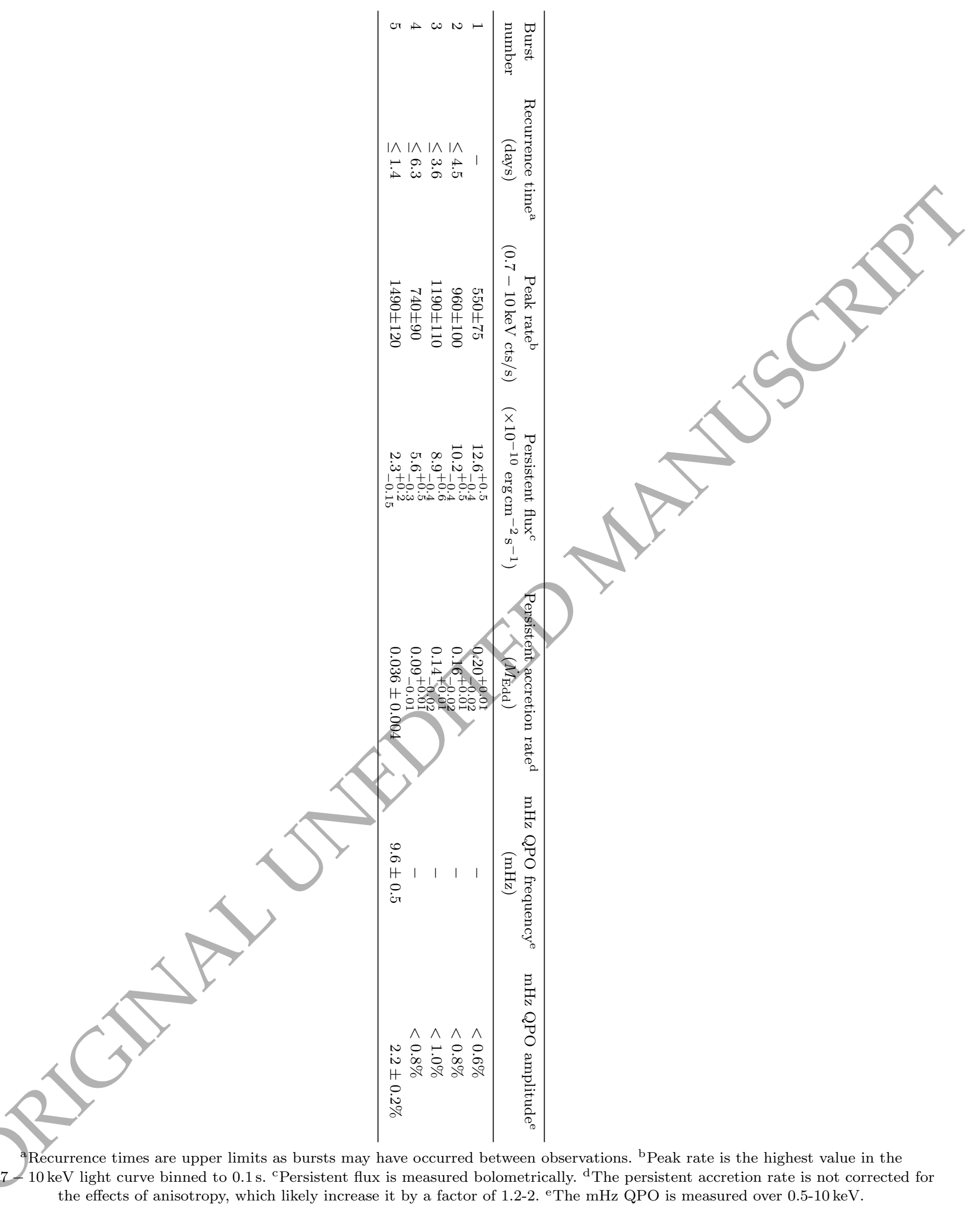




\section{AFFILIATIONS}

${ }^{1}$ Department of Physics and Astronomy, University of Southampton, Highfield, Southampton, SO17 1BJ

${ }^{2}$ Department of Astronomy, University of Maryland, College Park, MD 20742, USA

${ }^{3}$ NASA/Goddard Space Flight Center, Code 662, Greenbelt, MD 20771, USA

${ }^{4}$ Instituto Argentino de Radioastronomía(CCT-La Plata, CONICET; CICPBA), C.C. No. 5, 1894 Villa Elisa, Argentina

${ }^{5}$ Facultad de Ciencias Astronómicas y Geofísicas, Universidad Nacional de La Plata, Paseo del Bosque s/n, 1900 La Plata, Argentina

${ }^{6}$ Istanbul University, Science Faculty, Department of Astronomy and Space Sciences, Beyazit, 34119, Istanbul, Turkey

${ }^{7}$ Istanbul University Observatory Research and Application Center, Istanbul University 34119, Istanbul Turkey

${ }^{8}$ National Space Institute, Technical University of Denmark, Elektrovej 327-328, DK-2800 Lyngby, Denmark

${ }^{9}$ MIT Kavli Institute for Astrophysics and Space Research, Massachusetts Institute of Technology, Cambridge, MA 02139, USA

${ }^{10}$ IRAP, CNRS, UPS, CNES, 9 avenue du Colonel Roche, BP 44346, F-31028 Toulouse Cedex 4, France

${ }^{11}$ Eureka Scientific, Inc., 2452 Delmer Street, Oakland, CA 94602, USA

${ }^{12} \mathrm{SRON}$, Netherlands Institute for Space Research, Sorbonnelaan 2, 3584 CA Utrecht, The Netherlands

${ }^{13}$ Department of Astronomy, Tsinghua University, Shuangqing Road 30, Beijing 100084 China

${ }^{14}$ Tsinghua Center for Astrophysics, Tsinghua University, Shuangqing Road 30, Beijing 100084 China

${ }^{15}$ NASA Marshall Space Flight Center, NSSTC, 320 Sparkman Drive, Huntsville, AL 35805, USA

${ }^{16}$ Universities Space Research Association, Science and Technology Institute, 320 Sparkman Drive, Huntsville, AL 35805, USA

${ }^{17}$ Department of Astronomy, University of Michigan, 1085 South University Avenue, Ann Arbor, MI 48109-1104, USA

${ }^{18}$ Department of Astronomy \& Astrophysícs, Atatürk University, Erzurum, Turkey

${ }^{19}$ Astrophysics Science Division and Joint Space-Science Institute, NASA's Goddard Space Flight Center, Greenbelt, MD 20771, USA

${ }^{20}$ Department of Physics, Tor Yergata University of Rome, Via della Ricerca Scientifica 1, I-00133 Rome, Italy

${ }^{21}$ INAF - Astronomical Observatory of Rome, Via Frascati 33, I-00078 Monte Porzio Catone(Rome), Italy

${ }^{22}$ Space Sciences Laboratory, 7 Gauss Way, University of California, Berkeley, CA 94720-7450, USA

${ }^{23}$ Institute of Astronomy, Madingley Road, Cambridge, CB3 0HA 